\title{
Gene expression profiling of Duchenne muscular dystrophy reveals characteristics along disease progression
}

\author{
L.J. Tian, J.H. Cao, X.Q. Deng, C.L. Zhang, T. Qian, X.X. Song and B.S. Huang \\ Clinical Laboratory, Children's Hospital, Xuzhou, Jiangsu Province, China \\ Corresponding author: L.J. Tian \\ E-mail: tlj1168@163.com
}

Genet. Mol. Res. 13 (1): 1402-1411 (2014)

Received January 17, 2013

Accepted July 28, 2013

Published February 28, 2014

DOI http://dx.doi.org/10.4238/2014.February.28.13

\begin{abstract}
Duchenne muscular dystrophy (DMD) is the most common form of muscular dystrophy with no cure currently available. In this study, using two microarray data sets obtained from the Gene Expression Omnibus database, we conducted a dysfunctional pathway-enrichment analysis and investigated deregulated genes that are specific to different phases of the disease in order to determine pathogenic characteristics in the progression of DMD. We identified 41 and 33 dysfunctional pathways that were enriched with differentially expressed genes in presymptomatic patients and in symptomatic patients, respectively. Over $70 \%$ of pathways were shared between both phases and many of them involved the inflammatory process, suggesting that inflammatory cascades were induced soon after the birth of the patients. Further investigation showed that presymptomatic patients performed better with respect to muscle regeneration and cardiac muscle calcium homeostasis maintenance. Neuronal nitric oxide synthase, dihydropyridine receptors, sarcoplasmic/ endoplasmic reticulum calcium ATPase, and phospholamban may serve as potential targets for further molecular diagnostic tests. Our results may provide a better understanding for the treatment of DMD.
\end{abstract}

Key words: Duchenne muscular dystrophy; Dysfunctional pathway; Disease progression 


\section{INTRODUCTION}

Duchenne muscular dystrophy (DMD), which affects approximately 1 in 3500 live male births (Emery, 2002), is a severe and progressive disease characterized by muscle fiber degeneration and central nervous system disorders caused by mutations in the dystrophin gene located on the short arm of the X chromosome. As a 427-kDa cytoskeletal protein, dystrophin locates underneath the sarcolemma and assembles with sarcolemmal proteins to form the dystrophin-associated protein complex (DAPC), which includes dystroglycans, sarcoglycans, syntrophins, and sarcospan. The absence of or defects in dystrophin result in disruption of the DAPC, leading to chronic inflammation, progressive muscle degeneration, and replacement of muscle with fibroadipose tissue (Hoffman et al., 1987). DMD patients often lose independent ambulation by the time they reach 13 years of age (Hoffman et al., 1987), and generally die of respiratory failure in their late teens or early twenties (Blake et al., 2002). Cognitive defects and brain abnormalities are also important features of this disease and have been described previously (Anderson et al., 2002). Although abnormal muscle histologies are always present, DMD patients are otherwise clinically normal at birth. Initial symptoms of DMD are usually observed between the ages of 2 and 5 years (Dubowitz, 1978; Jennekens et al., 1991), with the child presenting delayed walking, frequent falls, or difficulty in running and climbing stairs. The first two years are thus considered to be clinically presymptomatic (Pescatori et al., 2007).

Since the discovery of the $D M D$ gene over 20 years ago (Koenig et al., 1987; Kunkel et al., 1987), numerous promising therapeutic strategies have been proposed. Nevertheless, all of these approaches have many hurdles to overcome and there is still no curative treatment for DMD. Although mutations of the dystrophin gene are primarily responsible for defects that underlie the pathology of DMD, other factors may also contribute importantly to the pathology. Secondary changes of DMD involve cytokines (De Pasquale et al., 2012), nitric oxide synthase (NOS; Altamirano et al., 2012), metabolic crisis (Chen et al., 2000), growth factors (Gehrig et al., 2012), calcium homeostasis (Head, 2010), and mast cell degranulation (Gorospe et al., 1994), suggesting that the pathological process of DMD is highly complicated and various biochemical pathways may contribute to its progression. Current available high throughput experimental strategies help in clarifying these pathogenic pathways and in identifying or modifying factors involved in DMD, thus constituting a great advance for the development or improvement of therapies.

We hypothesized that dysfunctional pathways are strongly related to specific pathogenic states and that dysfunctional pathways enriched with differentially expressed genes play more important roles in progression of the disease than those showing no enrichment pattern. Dynamic changes of dysfunctional pathways enriched with differentially expressed genes can capture pathogenic characteristics of corresponding stages and reflect the roles of those pathways in the progression of DMD. Here, using microarray gene expression data collected from the Gene Expression Omnibus (GEO) database for the two phases, we carried out pathway-enrichment analysis for the progression of DMD, and further investigated the deregulated genes specific to different phases in the hopes of providing new targets for future molecular diagnostic tests.

\section{MATERIAL AND METHODS}

\section{Microarray data}

In this study, two datasets (GSE6011 and GSE3307) from the GEO were used. They 
contained 19 presymptomatic samples, 8 symptomatic samples, and 14 controls. Sample information is listed in Table S1. Both datasets were based on the [HG-U133A] Affymetrix Human Genome U133A Array.

\section{Differentially expressed gene analysis}

Entire data sets, including CEL and simple omnibus format in text (SOFT)-formatted family files, were downloaded for all samples. Raw intensity values from CEL files were normalized by Robust Multi-array Analysis (RMA) (Irizarry et al., 2003) as follows. First, the effects of background noise and processing artifacts were neutralized with model-based background correction. Second, expression values were aligned to a common scale with quantile normalization. Third, data were summarized and a single expression value for each probe set was generated with an iterative median polishing procedure. The resulting RMA expression values ( $\log _{2}$-transformed) were derived by probe set level analysis from the raw data of CEL files. The $\log _{2}$-transformed RMA values for presymptomatic samples, symptomatic samples, and normal samples were stored separately to further identify significantly differentially expressed genes. The Student $t$-test with multiple test correction (Benjamini and Hochberg) was carried out for the presymptomatic-normal pair and symptomatic-normal pair, respectively, to detect differentially expressed genes. The threshold of significantly expressed genes was set to $\mathrm{P}<0.01$ in this study. Differentially expressed probe sets were identified using fold-change for up- or down-regulation. Differentially expressed genes in a specific pathway were combined, and were then hierarchically clustered to represent expression patterns using average linkage and Euclidean distance as a measurement of similarity. All of the above procedures were performed using the R statistical software (v2.14.1) with BioConductor and Limma packages (3.12.1) and libraries (Smyth et al., 2005).

\section{Pathway-enrichment analysis}

Selected probes were annotated according to the SOFT files. All genes were then mapped to Kyobo Encyclopedia of Genes and Genomes (KEGG) pathways (http://www.genome.jp/kegg/). Enrichment analysis was carried out by the hyper-geometric distribution test to identify pathways that were significantly enriched with differentially expressed genes. The observed class was the number of differentially expressed genes to the total number of genes for each family, while the expected class was the number of all differentially expressed genes to the total number of genes for all families.

\section{RESULTS}

The average fold-change of presymptomatic or symptomatic patients relative to controls for each gene indicated the gene expression level at the corresponding stage. Compared with normal controls, 1023 genes were identified as differentially expressed in presymptomatic patients. Among them, 747 showed up-regulation and 276 showed down-regulation. For symptomatic patients, 1407 genes showed a differential expression pattern compared to controls, including 977 up-regulated genes and 430 down-regulated genes. Detailed information for these genes is provided in Table S2.

Of all well-characterized human genes in the array, 5157 genes could be mapped to various pathways. For differentially expressed genes, 494 and 619 genes mapped to KEGG pathways for presymptomatic and symptomatic patients, respectively. According to the enrichment analysis, 
41 pathways were enriched with differentially expressed genes in presymptomatic patients, while 33 pathways showed enrichment with differentially expressed genes in symptomatic patients. Of these, 11 pathways showed the enrichment pattern in presymptomatic patients only (Table 1) and nine of them involved basic metabolism, including pathways for glycometabolism, lipometabolism, and proteometabolism. Three pathways were enriched with differentially expressed genes in symptomatic patients only, including bacterial invasion of epithelial cells, tight junction, D-glutamine, and D-glutamate metabolism. Thirty pathways were shared across both phases (Table 2), involving extracellular matrix homeostasis, energy metabolism, and inflammation. Furthermore, two $D M D$-related pathways, the dilated cardiomyopathy pathway (hsa05414) and the viral myocarditis pathway (hsa05416), were included in the shared pathways. Several KEGG pathways were related to other pathways and their relationships are illustrated in Figure 1. Results of the investigation of deregulated genes specific to different phases are illustrated in Figure 2.

Table 1. Pathways enriched with differentially expressed genes in presymptomatic phase only.

\begin{tabular}{llr}
\hline Pathway ID & Pathway name & P value \\
\hline hsa05168 & Herpes simplex infection & $1.14 \mathrm{E}-03$ \\
hsa04141 & Protein processing in endoplasmic reticulum & $1.88 \mathrm{E}-03$ \\
hsa04976 & Bile secretion & $2.42 \mathrm{E}-03$ \\
hsa00630 & Glyoxylate and dicarboxylate metabolism & $2.50 \mathrm{E}-03$ \\
hsa04971 & Gastric acid secretion & $3.27 \mathrm{E}-03$ \\
hsa05164 & Influenza A & $5.07 \mathrm{E}-03$ \\
hsa00360 & Phenylalanine metabolism & $5.10 \mathrm{E}-03$ \\
hsa00400 & Phenylalanine, tyrosine and tryptophan biosynthesis & $7.54 \mathrm{E}-03$ \\
hsa00010 & Glycolysis/gluconeogenesis & $7.73 \mathrm{E}-03$ \\
hsa00785 & Lipoic acid metabolism & $9.16 \mathrm{E}-03$ \\
hsa04640 & Hematopoietic cell lineage & $9.46 \mathrm{E}-03$ \\
\hline
\end{tabular}

Table 2. Pathways enriched with differentially expressed genes in both presymptomatic and symptomatic phases.

\begin{tabular}{|c|c|c|c|}
\hline Pathway ID & Pathway name & Presymptomatic P value & Symptomatic P value \\
\hline hsa04145 & Phagosome & $1.61 \mathrm{E}-11$ & $6.43 \mathrm{E}-12$ \\
\hline hsa05150 & Staphylococcus aureus infection & $1.21 \mathrm{E}-11$ & $9.07 \mathrm{E}-11$ \\
\hline hsa05416 & Viral myocarditis & $1.05 \mathrm{E}-11$ & $5.40 \mathrm{E}-10$ \\
\hline hsa05140 & Leishmaniasis & $1.33 \mathrm{E}-07$ & $2.73 \mathrm{E}-08$ \\
\hline hsa04142 & Lysosome & $2.85 \mathrm{E}-05$ & 8.07E-08 \\
\hline hsa04514 & Cell adhesion molecules (CAMs) & $6.49 \mathrm{E}-08$ & $1.25 \mathrm{E}-07$ \\
\hline hsa05130 & Pathogenic Escherichia coli infection & 3.05E-09 & $1.71 \mathrm{E}-07$ \\
\hline hsa05145 & Toxoplasmosis & $8.68 \mathrm{E}-07$ & $5.77 \mathrm{E}-07$ \\
\hline hsa05310 & Asthma & $2.62 \mathrm{E}-07$ & $6.21 \mathrm{E}-07$ \\
\hline hsa04612 & Antigen processing and presentation & $8.52 \mathrm{E}-12$ & $1.20 \mathrm{E}-06$ \\
\hline hsa04672 & Intestinal immune network for IgA production & $4.61 \mathrm{E}-07$ & $2.28 \mathrm{E}-06$ \\
\hline hsa05152 & Tuberculosis & $6.64 \mathrm{E}-09$ & $3.88 \mathrm{E}-06$ \\
\hline hsa05330 & Allograft rejection & $2.12 \mathrm{E}-10$ & $1.42 \mathrm{E}-05$ \\
\hline hsa04510 & Focal adhesion & $2.24 \mathrm{E}-07$ & $3.43 \mathrm{E}-05$ \\
\hline hsa04974 & Protein digestion and absorption & $5.59 \mathrm{E}-04$ & $4.05 \mathrm{E}-05$ \\
\hline hsa05332 & Graft-versus-host disease & $1.05 \mathrm{E}-09$ & $4.12 \mathrm{E}-05$ \\
\hline hsa04512 & ECM-receptor interaction & $2.27 \mathrm{E}-08$ & $7.30 \mathrm{E}-05$ \\
\hline hsa04940 & Type I diabetes mellitus & $2.85 \mathrm{E}-09$ & $7.82 \mathrm{E}-05$ \\
\hline hsa05133 & Pertussis & $1.21 \mathrm{E}-05$ & $8.65 \mathrm{E}-05$ \\
\hline hsa05323 & Rheumatoid arthritis & $4.45 \mathrm{E}-04$ & 4.38E-04 \\
\hline hsa05320 & Autoimmune thyroid disease & $1.69 \mathrm{E}-07$ & $1.02 \mathrm{E}-03$ \\
\hline hsa04540 & Gap junction & $6.03 \mathrm{E}-03$ & $2.05 \mathrm{E}-03$ \\
\hline hsa04610 & Complement and coagulation cascades & $4.55 \mathrm{E}-05$ & $2.19 \mathrm{E}-03$ \\
\hline hsa04810 & Regulation of actin cytoskeleton & $4.29 \mathrm{E}-04$ & $5.84 \mathrm{E}-03$ \\
\hline hsa05146 & Amoebiasis & $1.27 \mathrm{E}-04$ & $6.53 \mathrm{E}-03$ \\
\hline hsa04670 & Leukocyte transendothelial migration & $1.63 \mathrm{E}-06$ & $7.32 \mathrm{E}-03$ \\
\hline hsa05322 & Systemic lupus erythematosus & $1.50 \mathrm{E}-04$ & $8.29 \mathrm{E}-03$ \\
\hline hsa05169 & Epstein-Barr virus infection & $3.53 \mathrm{E}-04$ & $8.66 \mathrm{E}-03$ \\
\hline hsa05414 & Dilated cardiomyopathy & $5.36 \mathrm{E}-03$ & $9.47 \mathrm{E}-03$ \\
\hline hsa05166 & HTLV-I infection & $1.07 \mathrm{E}-04$ & $9.63 \mathrm{E}-03$ \\
\hline
\end{tabular}



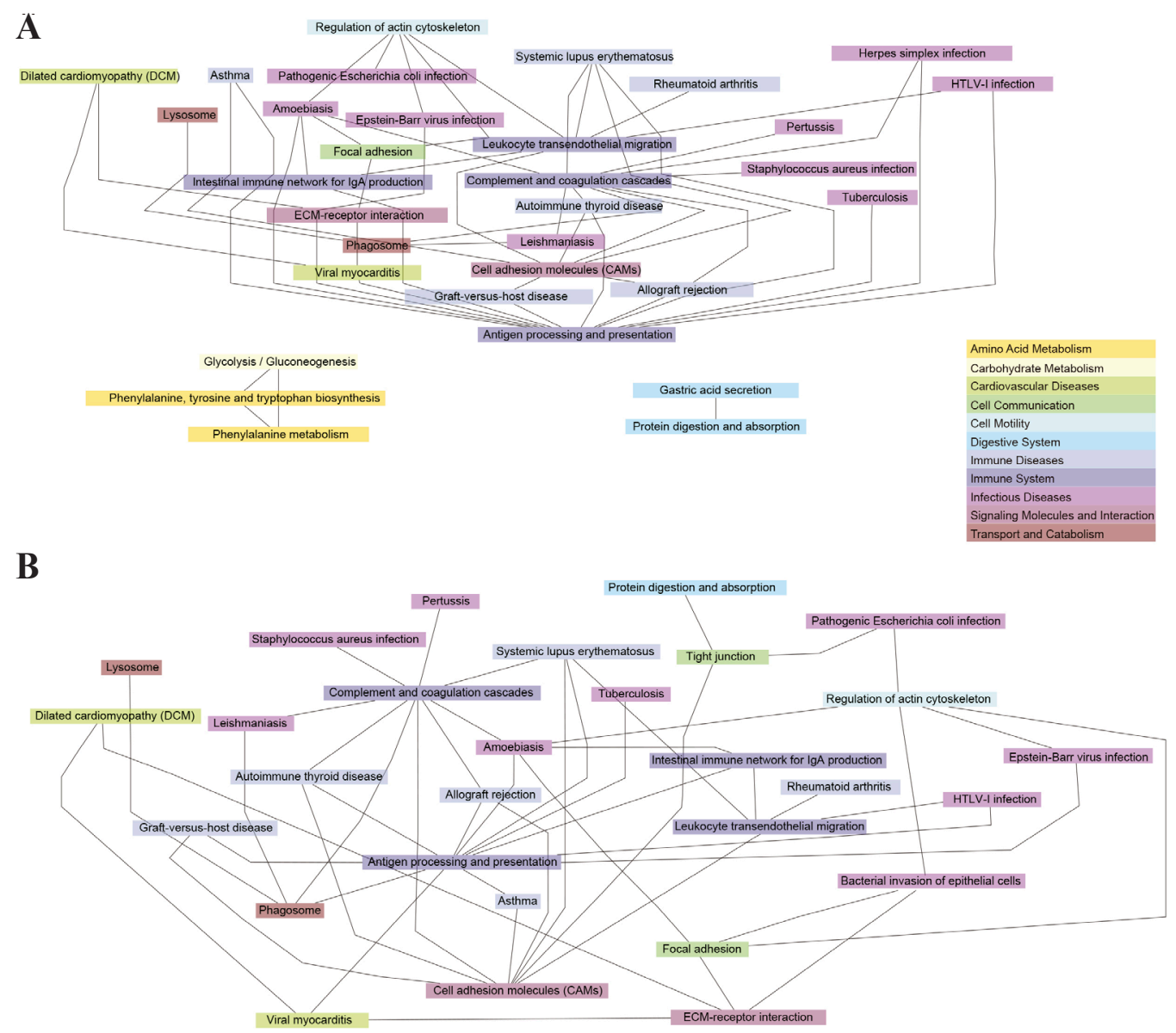

Figure 1. Relationship of KEGG biological pathways enriched with differentially expressed genes in presymptomatic (A) and symptomatic patients (B). For each phase, pathways not related with other pathways according to the KEGG database were not showed. Category of each pathway is shown in the right bar.

\section{DISCUSSION}

The progressive pathophysiology of DMD is a highly complex process. Secondary changes are caused by mutations of the dystrophin gene, including disruptions of sarcolemma, activation of the calcium-dependent degradative pathway due to flux of extracellular $\mathrm{Ca}^{2+}$, muscle necrosis, or apoptosis (Turner et al., 1993), along with proliferation of connective and adipose tissue and infiltration of immune cells (Blake et al., 2002). Although these downstream deregulated pathways may contribute to the pathology of DMD, they are not sufficient to explain it. Insights into these processes may be gleaned from gene expression profile analysis, which allows examination of changes in many mRNA levels and reveals groups of deregulated genes and dysfunctional pathways. Dysfunctional pathways enriched with differentially expressed genes are thought to play more important roles in the progression of DMD than those showing no enrichment pattern. Here, using two datasets from the GEO database, we 


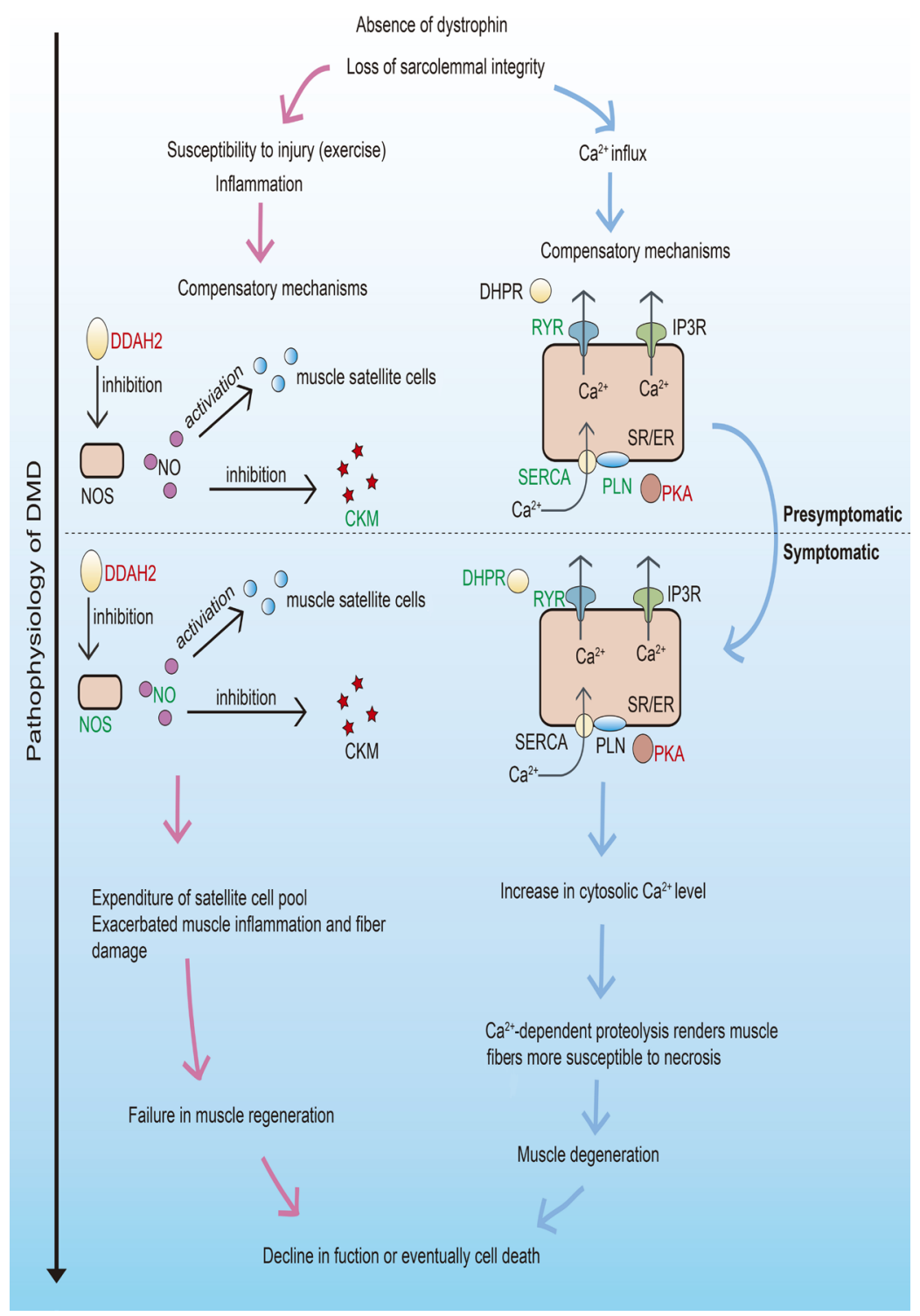

Figure 2. Schematic representation for the pathophysiology of DMD. Differentially expressed genes related with muscle regeneration and calcium homeostasis are shown. Proteins encoded by overexpression genes are shown in red and proteins encoded by down-regulation genes are shown in green. Proteins shown in black means that no differential gene expression was detected. DHPR is specific in cardiac muscle. In cardiac muscle, the RYR gene (RYR2) did not show deregulation in both phases. DDAH2: dimethylarginine dimethylaminohydrolase 2, encoded by $D D A H 2$; NOS: nitric oxide synthase 1 (neuronal), encoded by $N O S 1$; $\mathrm{NO}=$ nitric oxide; $\mathrm{CKM}=$ creatine kinase, muscle, encoded by $C K M$; PLN = phospholamban, encoded by $P L N$; SERCA = sarcoplasmic/endoplasmic reticulum calcium ATPase, encoded by $A T P 2 A 1$ and $A T P 2 A 2$; PKA = protein kinase A, related gene: $P R K A C B$; $\mathrm{RYR}=$ ryanodine receptors, related gene: $R Y R 1 ; \mathrm{DHPR}=$ dihydropyridine receptors, deregulated in cardiac muscle only, related gene: $C A C N A 1 B$ and $C A C N A 1 D$. 
provided dysfunctional pathways enriched with differentially expressed genes and illustrated their interactions in both the presymptomatic and symptomatic phases of DMD.

Results showed that the two phases shared most of the pathways (over 70\%), suggesting that many abnormal pathways are always present, whether or not patients are phenotypically indistinguishable from normal patients. As shown in Figure 1, most of the deregulated pathways involved inflammatory/immune responses, including immune disease, immune system, and infectious disease. These immune/inflammatory pathways mainly indicated the infiltration of immune cells, such as T cells (McDouall et al., 1990; Spencer et al., 1997), macrophages (McDouall et al., 1990; Spencer et al., 1997), eosinophils (Cai et al., 2000), and mast cells (Granchelli et al., 1995; Nahirney et al., 1997), into muscles and elevated levels of various inflammatory cytokines. A previous study showed that in vivo depletions of CD4 or CD8 T cells (Spencer et al., 2001) or macrophages (Wehling et al., 2001) significantly reduced the pathology in mdx mice, and that the efficacy of the only drugs currently available for the treatment of DMD (glucocorticoids) in delaying DMD progression is mainly based on the suppression of inflammation (Serra et al., 2012), implicating the important roles of immune/ inflammation responses in aggravating the disease. Consistent with previous reports (Chen et al., 2005), the sharing of these inflammatory pathways across both stages of DMD suggested that inflammatory cascades were induced soon after the birth of the patients. However, some genes in symptomatic patients did show much higher or lower expression than those in the presymptomatic patients, implying that their underlying differences may contribute to different clinical manifestations.

Loss of sarcolemmal integrity due to the absence of dystrophin leads to susceptibility to muscle injury, resulting in inflammation and muscle regeneration through the activation of muscle satellite cells. In addition, previous studies (Franco Jr. and Lansman, 1990; Turner et al., 1993; Hopf et al., 1996; De Backer et al., 2002; Blake et al., 2002) have demonstrated the high influx of extracellular calcium through a dystrophin-deficient membrane, which may lead to the activation of calpains and subsequent muscle necrosis or apoptosis along with the inflammation response. Thus, to capture the characteristics of each phase, we further focus on mechanisms related to muscle regeneration and calcium homeostasis.

With respect to muscle regeneration, the fibrosis process may not change substantially during DMD deterioration since up-regulation of the primary fibril-forming collagens (types I and III) and additional fibrillar collagens (type V) was observed in both stages. Furthermore, cathepsin $\mathrm{K}$, a member of the papain family of cystein proteases that is responsible for the degradation of collagen type I, which may serve as a potential attempt to counteract collagen deposition, was also overexpressed in both presymptomatic and symptomatic patients. However, as shown in Figure 2, a decrease of NOS (NOS1) mRNA expression was found only in symptomatic patients. Reduction in this enzyme results in reduced production of the free radical nitric oxide, which is a free radical that protects the muscle against oxidative injury (NiebrójDobosz and Hausmanowa-Petrusewicz, 2005) and activates muscle satellite cells (Anderson, 2000). A previous study (Wehling et al., 2001) demonstrated that normal levels of NOS in muscle reduced macrophage concentrations in mdx mouse muscles, substantially preventing muscle membrane injury and decreasing serum creatine kinase concentrations. The massive release of creatine kinase would cause a respondent inflammatory response. We observed that creatine kinase in muscle showed down-regulation only in presymptomatic patients, suggesting that NOS functions normally in presymptomatic patients, functioning to activate regular muscle regeneration and to prevent further muscle damage. Thus, deregulation of NOS may 
contribute to the progression of DMD. In addition, increased expression in dimethylarginine dimethylaminohydrolase 2 (DDAH2), which metabolizes asymmetric methylarginines, which in turn act to inhibit NOS, was observed in both phases. Its fold-change in symptomatic patients (0.55-0.58) was slightly higher than that in presymptomatic patients $(0.37-0.46)$, suggesting that down-regulation of NOS may be due to the increasing expression of DDAH2. Further investigation of the detailed regulatory mechanism is needed for confirmation of this hypothesis.

With respect to calcium homeostasis, along with the influx of extracellular calcium through the dystrophin-deficient membrane, uptake of calcium by the sarcoplasmic reticulum (SR) Ca-ATPase pump and/or extrusion by the sodium/calcium exchanger (NCX) and the plasma membrane $\mathrm{Ca}^{2+}$-ATPase (PMCA) may help to reduce the calcium concentration. Here, genes related with NCX and PMCA did not show differential expression at either phase. However, as shown in Figure 2, genes encoding the sarcoplasmic/endoplasmic reticulum calcium ATPase (SERCA) in cardiac muscle and phospholamban (PLN) showed downregulation in presymptomatic patients and the PLN/SERCA ratio was actually decreased since the fold-change of PLN (-1.00419) was almost twice as much as that of SERCA (ATP2A1: -0.50584; ATP2A2: -0.55769). SERCA activity is physiologically modulated by PLN, which, in its dephosphorylated form, tightly interacts with SERCA and inhibits its activity (MacLennan and Kranias, 2003). Phosphorylation of PLN by protein kinase A (PKA), undoes SERCA inhibition, increasing its affinity for $\mathrm{Ca}^{2+}$ and accelerating its activity. A decreased PLN to SERCA ratio may also facilitate the uptake of calcium of SERCA from the cytoplasm to the SR (Haghighi et al., 2004). Thus, in presymptomatic patients, the overexpression of PKA-related genes and a decreased PLN/SERCA ratio may accelerate the phosphorylation of PLN and remove SERCA inhibition, thus promoting the flux of calcium from the cytoplasm to the SR, and reducing the calcium level in the cytoplasm. Meanwhile, the CACNAID and $C A C N A 1 B$ genes only showed down-regulation in symptomatic patients. Proteins encoded by these genes are subunits of dihydropyridine receptors (DHPR), which couple with ryanodine receptors (RYR) and suppress spontaneous SR elementary $\mathrm{Ca}^{2+}$ release events (Teichmann et al., 2008). Down-regulation of $C A C N A 1 D$ and $C A C N A 1 B$ may relieve inhibitory control of DHPR, contributing to increased $\mathrm{Ca}^{2+}$ levels in the cytoplasm. The RYR gene in skeletal muscle (RYRl) was down-regulated, while the RYR gene in cardiac muscle (RYR2) was not differentially expressed in the two phases. PKA was also up-regulated in symptomatic patients, but the affinity of SERCA for $\mathrm{Ca}^{2+}$ observed here might be weaker than that of the presymptomatic patients since the PLN/SERCA ratio did not change. In short, calcium homeostasis in the cardiac muscle may be better maintained in presymptomatic patients by the influx of extracellular calcium through the dystrophin-deficient membrane. Most DMD patients suffer from dilated cardiomyopathy. Therefore, DHPR and PLN/SERCA may serve as potential targets for ameliorating their pathogenesis.

In summary, we conducted a pathway-enrichment analysis in both presymptomatic and symptomatic DMD patients to capture pathogenic characteristics related to progression of the disease. Results showed that the two stages shared many inflammatory pathways, suggesting that inflammatory cascades were induced soon after the birth of the patients. With respect to specific characteristics of each phase, presymptomatic patients performed better in muscle regeneration and cardiac muscle calcium homeostasis maintenance. Our results may provide a better understanding of DMD and further contribute information of potential targets that can be used for molecular diagnostic tests. 


\section{ACKNOWLEDGMENTS}

Research supported by the Planning Program of Xuzhou Science and Technology Bureau in Jiangsu, China (\#Y3014).

\section{Supplementary material}

\section{REFERENCES}

Altamirano F, López JR, Henríquez C, Molinski T, et al. (2012). Increased resting intracellular calcium modulates NFผB-dependent inducible nitric-oxide synthase gene expression in dystrophic $m d x$ skeletal myotubes. J. Biol. Chem. 287: 20876-20887.

Anderson JE (2000). A role for nitric oxide in muscle repair: nitric oxide-mediated activation of muscle satellite cells. Mol. Biol. Cell 11: 1859-1874.

Anderson JL, Head SI, Rae C and Morley JW (2002). Brain function in Duchenne muscular dystrophy. Brain 125: 4-13.

Blake DJ, Weir A, Newey SE and Davies KE (2002). Function and genetics of dystrophin and dystrophin-related proteins in muscle. Physiol. Rev. 82: 291-329.

Cai B, Spencer MJ, Nakamura G, Tseng-Ong L, et al. (2000). Eosinophilia of dystrophin-deficient muscle is promoted by perforin-mediated cytotoxicity by T cell effectors. Am. J. Pathol. 156: 1789-1796.

Chen YW, Zhao P, Borup R and Hoffman EP (2000). Expression profiling in the muscular dystrophies: identification of novel aspects of molecular pathophysiology. J. Cell Biol. 151: 1321-1336.

Chen YW, Nagaraju K, Bakay M, McIntyre O, et al. (2005). Early onset of inflammation and later involvement of TGFbeta in Duchenne muscular dystrophy. Neurology 65: 826-834.

De Backer F, Vandebrouck C, Gailly P and Gillis JM (2002). Long-term study of $\mathrm{Ca}^{2+}$ homeostasis and of survival in collagenase-isolated muscle fibres from normal and $m d x$ mice. J. Physiol. 542: 855-865.

De Pasquale L, D’Amico A, Verardo M, Petrini S, et al. (2012). Increased muscle expression of interleukin-17 in Duchenne muscular dystrophy. Neurology 78: 1309-1314.

Dubowitz V (1978). Muscle disorders in childhood. Major Probl. Clin. Pediatr. 16: iii-282.

Emery AE (2002). The muscular dystrophies. Lancet 359: 687-695.

Franco A Jr and Lansman JB (1990). Calcium entry through stretch-inactivated ion channels in mdx myotubes. Nature 344: 670-673.

Gehrig SM, van der Poel C, Hoeflich A, Naim T, et al. (2012). Therapeutic potential of PEGylated insulin-like growth factor I for skeletal muscle disease evaluated in two murine models of muscular dystrophy. Growth Horm. IGF Res. 22: 69-75.

Gorospe JR, Tharp MD, Hinckley J, Kornegay JN, et al. (1994). A role for mast cells in the progression of Duchenne muscular dystrophy? Correlations in dystrophin-deficient humans, dogs, and mice. J. Neurol. Sci. 122: 44-56.

Granchelli JA, Pollina C and Hudecki MS (1995). Duchenne-like myopathy in double-mutant $m d x$ mice expressing exaggerated mast cell activity. J Neurol. Sci. 131: 1-7.

Haghighi K, Gregory KN and Kranias EG (2004). Sarcoplasmic reticulum Ca-ATPase-phospholamban interactions and dilated cardiomyopathy. Biochem. Biophys. Res. Commun. 322: 1214-1222.

Head SI (2010). Branched fibres in old dystrophic $m d x$ muscle are associated with mechanical weakening of the sarcolemma, abnormal $\mathrm{Ca}^{2+}$ transients and a breakdown of $\mathrm{Ca}^{2+}$ homeostasis during fatigue. Exp. Physiol. 95: 641-656.

Hoffman EP, Brown RH Jr and Kunkel LM (1987). Dystrophin: the protein product of the Duchenne muscular dystrophy locus. Cell 51: 919-928.

Hopf FW, Turner PR, Denetclaw WF Jr, Reddy P, et al. (1996). A critical evaluation of resting intracellular free calcium regulation in dystrophic $m d x$ muscle. Am. J. Physiol. 271: C1325-C1339.

Irizarry RA, Hobbs B, Collin F, Beazer-Barclay YD, et al. (2003). Exploration, normalization, and summaries of high density oligonucleotide array probe level data. Biostatistics 4: 249-264.

Jennekens FG, ten Kate LP, de Visser M and Wintzen AR (1991). Diagnostic criteria for Duchenne and Becker muscular dystrophy and myotonic dystrophy. Neuromuscul. Disord. 1: 389-391.

Koenig M, Hoffman EP, Bertelson CJ, Monaco AP, et al. (1987). Complete cloning of the Duchenne muscular dystrophy (DMD) cDNA and preliminary genomic organization of the DMD gene in normal and affected individuals. Cell 50: 509-517. 
Kunkel LM, Monaco AP, Hoffman E, Koenig M, et al. (1987). Molecular studies of progressive muscular dystrophy (Duchenne). Enzyme 38: 72-75.

MacLennan DH and Kranias EG (2003). Phospholamban: a crucial regulator of cardiac contractility. Nat. Rev. Mol. Cell Biol. 4: 566-577.

McDouall RM, Dunn MJ and Dubowitz V (1990). Nature of the mononuclear infiltrate and the mechanism of muscle damage in juvenile dermatomyositis and Duchenne muscular dystrophy. J. Neurol. Sci. 99: 199-217.

Nahirney PC, Dow PR and Ovalle WK (1997). Quantitative morphology of mast cells in skeletal muscle of normal and genetically dystrophic mice. Anat. Rec. 247: 341-349.

Niebrój-Dobosz I and Hausmanowa-Petrusewicz I (2005). The involvement of oxidative stress in determining the severity and progress of pathological processes in dystrophin-deficient muscles. Acta Biochim. Pol. 52: 449-452.

Pescatori M, Broccolini A, Minetti C, Bertini E, et al. (2007). Gene expression profiling in the early phases of DMD: a constant molecular signature characterizes DMD muscle from early postnatal life throughout disease progression. FASEB J. 21: 1210-1226.

Serra F, Quarta M, Canato M and Toniolo L (2012). Inflammation in muscular dystrophy and the beneficial effects of nonsteroidal anti-inflammatory drugs. Muscle Nerve 46: 773-784.

Smyth GK, Michaud J and Scott HS (2005). Use of within-array replicate spots for assessing differential expression in microarray experiments. Bioinformatics 21: 2067-2075.

Spencer MJ, Walsh CM, Dorshkind KA, Rodriguez EM, et al. (1997). Myonuclear apoptosis in dystrophic $m d x$ muscle occurs by perforin-mediated cytotoxicity. J. Clin. Invest. 99: 2745-2751.

Spencer MJ, Montecino-Rodriguez E, Dorshkind K and Tidball JG (2001). Helper (CD4(+)) and cytotoxic (CD8(+)) T cells promote the pathology of dystrophin-deficient muscle. Clin. Immunol. 98: 235-243.

Teichmann MD, Wegner FV, Fink RH, Chamberlain JS, et al. (2008). Inhibitory control over $\mathrm{Ca}^{2+}$ sparks via mechanosensitive channels is disrupted in dystrophin deficient muscle but restored by mini-dystrophin expression. PloS One 3: e3644.

Turner PR, Schultz R, Ganguly B and Steinhardt RA (1993). Proteolysis results in altered leak channel kinetics and elevated free calcium in $m d x$ muscle. J. Membrane Biol. 133: 243-251.

Wehling M, Spencer MJ and Tidball JG (2001). A nitric oxide synthase transgene ameliorates muscular dystrophy in $m d x$ mice. J. Cell Biol. 155: 123-131. 